\title{
DESIDRATAÇÃO OSMÓTICA DO JENIPAPO (Genipa americana L.) ${ }^{1}$
}

\author{
Samara Alvachian Cardoso ANDRADE ${ }^{2 *}$, Juliana Cavalcante METRI ${ }^{3}$,
}

\author{
Benício de BARROS NETO ${ }^{4}$, Nonete Barbosa GUERRA ${ }^{5}$
}

\section{RESUMO}

O processo de desidratação osmótica foi aplicado ao Jenipapo (Genipa americana L.), para reduzir as perdas pós-colheita, diversificar o seu aproveitamento industrial e obter um produto com boas características organolépticas e boa estabilidade microbiológica. Os frutos foram adquiridos na feira livre de Afogados, na região metropolitana do Recife, nas condições em que são comercializados, subdivididos em cubos e submetidos a diversas condições de processamento, segundo planejamento fatorial $2^{3}$ de modo a determinar a influência das variáveis independentes: temperatura, tipo de açúcar e concentração do agente osmótico sobre a perda de umidade, ganho de açúcar e redução de peso. O processo foi complementado por secagem em estufa, com ventilação forçada a $60^{\circ} \mathrm{C} \pm 5^{\circ} \mathrm{C}$, por $3 \mathrm{~h} \pm 10^{\prime}$, seguida do acondicionamento do produto em embalagem de polietileno e armazenamento à temperatura ambiente $\left(28^{\circ} \mathrm{C} \pm 3^{\circ} \mathrm{C}\right)$, por 90 dias. $\mathrm{Os}$ resultados demonstraram que: os produtos obtidos apresentaram significativa incorporação de sólidos; nenhuma das respostas foi influenciada pelo tipo de açúcar empregado, diferentemente da concentração do agente osmótico que além de refletir sobre todas elas, interagiu com a temperatura influindo sobre a perda de umidade; durante o armazenamento, os produtos apresentaram-se microbiologicamente estáveis, sem diferenças significativas, no que concerne às características organolépticas, embora os escores demonstrem uma preferência pelo produto obtido a partir de $60^{\circ} \mathrm{Brix}, 60^{\circ} \mathrm{C}$ e mistura de açúcar demerara e cristal. A elevada incorporação de sólidos ocorrida aponta para necessidade de proceder à otimização do produto com vista a sua redução.

Palavras-chave: desidratação osmótica; jenipapo; conservação.
\end{abstract}

\section{SUMMARY}

OSMOTIC DEHYDRATION OF JENIPAPO (Genipa americana L.). The process of osmotic dehydration was applied in order to reduce the losses post-harvest, to diversify the industrial utilization of the jenipapo (Genipa americana L.), and to obtain a product with good organoleptic characteristic and good microbiological stability. The fruits were purchased in the street fair of Afogados, a district of Recife, in the conditions in which they are sold. They were cut in cubes and submitted to several conditions of processing, through a factorial design $2^{3}$ to determine the influence of independent variables: temperature, kind of sugar and osmotic agent concentration over moisture loss, sugar uptake and weight reduction. The process was complemented with drying in stove, with forced ventilation at $60^{\circ} \mathrm{C} \pm 5^{\circ} \mathrm{C}$ for $3 \mathrm{~h} \pm 10^{\prime}$, after which the products were packed in polyethylene packaging and stored at room temperature $\left(28^{\circ} \mathrm{C} \pm 3^{\circ} \mathrm{C}\right)$ for ninety days. The results demonstrated that: a significant solids uptake occurred; none of the obtained responses was influenced by the type of sugar used, whereas the osmotic agent concentration not only reflected on all of them it also interacted with the temperature influencing over moisture loss; during storage time, the products were microbiologically stable, without significant differences, in the organoleptic caracteristic, although the scores have showed a preference for the products obtained under the $60^{\circ} \mathrm{Brix}, 60^{\circ} \mathrm{C}$ and a blend of demerara and crystal sugar. The high solids uptake occurred points to a necessity to achieve the optimization of the products in order to reduce it Keywords: osmotic dehydration; jenipapo; conservation.

\section{1 - INTRODUÇÂO}

O jenipapeiro (Genipa americana L.), pertencente à família "Rubiaceae", é considerado uma espécie vegetal de importância econômica, tanto pela sua essência florestal; quanto pela utilização dos seus frutos na produção de alimentos [Barros, 1970 apud 13, 14, 17].

A exemplo da maioria das frutas tropicais, seu fruto é altamente perecivel, deteriorando-se em poucos dias, fato que dificulta a sua comercialização, aumentando as perdas. Estima-se que, nas áreas tropicais e subtropicais, as perdas pós-colheitas de frutas e hortaliças oscilem entre $15 \%$ a $50 \%$, principalmente por manuseio e preservação inadequados (Chitarra e Chitarra, 1990 citado por [3]).

\footnotetext{
Recebido para publicação em 04/06/2002. Aceito para publicação em 21/11/2002 (000838)

2. Universidade Federal de Pernambuco - Depto. Eng. Quimica. E-mail samaraandrade@uol.com.br

3. Universidade Federal de Pernambuco, Depto. de Nutrição.

4. Universidade Federal de Pernambuco - Depto. de Química Fundamental.

5. Universidade Federal de Pernambuco, Depto. de Nutrição, LEAAL, R. Prof. Nelson Chaves, s/n, Campus Universitário CEP 50670-901, Recife - PE, Brasil.

* A quem a correspondência deve ser enviada.
}

Este fruto, raramente consumido in natura, tem sido utilizado de forma artesanal como matéria-prima para produção de compotas, cristalizados, sorvetes, refrescos, licor e vinho, é constituído por uma casca mole, parda ou pardacenta - amarelada, membranosa, fina e enrugada, que representa $9,56 \%$ do total do fruto; $73,81 \%$ de polpa com odor característico, muito forte, sabor doce acidulado, envolvendo numerosas sementes achatadas $(16,63 \%),[9,12,17,35]$.

A industrialização surge, portanto, como alternativa para reduzir as perdas, principalmente no pico da safra quando alcançam menores preços pelo excesso de oferta e pela concorrência de outras frutas.

Dos diversos processos para conservação de alimentos desenvolvidos pelo homem, a secagem, o mais antigo deles, apresenta-se viável para reverter este quadro.

Dos processos de secagem destaca-se a desidratação osmótica que acarreta a remoção da água, por diferença de gradiente, modificando a interação desta com demais compostos, de forma a reduzir sua disponibilidade para deterioração química e biológica [10, 24, 28, 36]. Devido à diferença de concentração entre o agente osmótico e a fruta, são criados dois fluxos simultâneos 
em contra corrente, através das paredes celulares: um de água que sai da fruta para a solução - o mais importante do ponto de vista da desidratação - e outro de soluto (sal ou açúcar) da solução para a fruta [20, 23, 29, 32, 36]. Conforme TORREGGIANI [36], há uma dupla transformação na fruta, que poderá resultar em redução de peso. Diante desta constatação foi realizado este trabalho com objetivo de obter um produto desidratado, osmoticamente, com boas características organolépticas e boa estabilidade microbiológica.

\section{2 - MATERIAL E METODOS}

\section{1 - Material}

Foi constituído por 11 lotes de aproximadamente 1200 g de Jenipapos (Genipa americana L.), adquiridos na feira livre de Afogados, na cidade do Recife, PE, no estágio de maturação em que são normalmente comercializados. Como agente osmótico utilizou-se a sacarose comercial (açúcar cristal), o açúcar demerara, metabissulfito de sódio e o ácido cítrico, ambos da MERCK, como conservante e acidulante respectivamente.

\section{2 - Delineamento}

Foi utilizado um planejamento fatorial $2^{3}$, acrescido de um ponto central, com as seguintes variáveis independentes: 1-Temperatura; 2-Concentração do agente osmótico; e 3- Tipo de agente osmótico. O planejamento consistiu em 11 ensaios, dos quais os três últimos são referentes a repetição do ponto central, cujas condições (niveis codificados e decodificados) encontram-se apresentado nas Tabelas 1 e 2, respectivamente [27]. O tempo de imersão de 4 horas foi estabelecido através de ensaios preliminares.

As respostas de interesse foram assim definidas:

Incorporação de sólidos (Ganho de açúcar) - calculada através de um balanço de massa de sólidos do processo:

IS $(\%)=100 \times(\mathrm{Bf} \mathrm{Mf}-\mathrm{Bi} \mathrm{Mi}) / \mathrm{Mi}$

Perda de Umidade - com base no peso inicial do material:

$\mathrm{PU}(\%)=100 \times(\mathrm{Ui} \mathrm{Mi}-\mathrm{Uf} \mathrm{Mf}) / \mathrm{Mi}$

Variação de peso - Calculada pela seguinte equação:

$\mathrm{VP}(\%)=100 \times(\mathrm{Mi}-\mathrm{Mf}) / \mathrm{Mi}$

\section{Sendo:}

$\mathrm{VP}=$ variação percentual de peso do material desidratado; $\mathrm{Mi}=$ massa inicial;

$\mathrm{Mf}=$ massa final;
$\mathrm{PU}=$ perda de umidade;

$\mathrm{Ui}=$ teor inicial de umidade $(\%)$;

$\mathrm{Uf}=$ teor final de umidade $(\%)$;

IS=incorporação de sólidos;

$\mathrm{Bi}=$ teor inicial de sólidos solúveis contidos no material (\%); $\mathrm{Bf}=$ teor final de sólidos solúveis contidos no material (\%).

TABELA 1. Niveis codificados das variáveis para os ensaios.

\begin{tabular}{cccc}
\hline$N^{\circ}$ de ensaio & ${ }^{\circ}$ Brix & Açúcar & Temperatura \\
\hline 1 & -1 & -1 & -1 \\
2 & 1 & -1 & -1 \\
3 & -1 & 1 & -1 \\
4 & 1 & 1 & -1 \\
5 & -1 & -1 & 1 \\
6 & 1 & -1 & 1 \\
7 & -1 & 1 & 1 \\
8 & 1 & 1 & 1 \\
9 & 0 & 0 & 0 \\
10 & 0 & 0 & 0 \\
11 & 0 & 0 & 0 \\
\hline
\end{tabular}

TABELA 2. Níveis decodificados das variáveis.

\begin{tabular}{cccc}
\hline \multicolumn{4}{c}{ Níveis decodificados } \\
\hline Niveis codificados & Temperatura $\left({ }^{\circ} \mathrm{C}\right)$ & $\begin{array}{c}\text { Concentração da } \\
\text { solução ('Brix) }\end{array}$ & $\begin{array}{c}\text { Tipo de agente } \\
\text { osmótico }\end{array}$ \\
\hline-1 & 50 & 50 & Demerara \\
0 & 60 & 60 & Demerara/Cristal \\
1 & 70 & 70 & Cristal \\
\hline
\end{tabular}

\section{3 - Processamento}

O processamento consistiu basicamente em: 1- recebimento da matéria-prima, pesagem e verificação dos aspectos gerais; 2- seleção segundo o grau de maturação e alterações visiveis; 3- lavagem em água potável, contendo 20 ppm de cloro por 20'; 4- descascamento realizado manualmente com faca de aço inoxidável; 5- corte em pedaços na forma de cubos de aproximadamente $10 \mathrm{~mm}$ de face; 6- inativação enzimática em solução aquosa de metabissulfito de sódio a $280 \mathrm{ppm}$ a $100^{\circ} \mathrm{C}$, por 5'; 7 - transferência da amostra para uma cuba contendo a solução osmótica acrescida de ácido cítrico para obter um pH entre 4 e 5; 8- drenagem da solução osmótica; 9- secagem do material em estufa com circulação de ar a $60^{\circ} \mathrm{C} \pm 5^{\circ} \mathrm{C}$ por aproximadamente $3 \mathrm{~h} \pm 10$; 10 - acondicionamento em embalagem de polietileno com capacidade de 500g; 11 - armazenados sob temperatura ambiente $28^{\circ} \mathrm{C}$ por 90 dias. 


\section{4- Determinações analíticas}

Antes e após o processo, realizaram-se determinações: 1- massa da amostra, em balança analitica; 2- sólidos solúveis em refratômetro de bancada BAUSCH e LOMB; 3- umidade em estufa a $105^{\circ} \mathrm{C}$, até peso constante.

\section{5 - Análise dos resultados}

Com vistas à otimização do processo, as respostas obtidas para todos os ensaios foram avaliadas quanto aos efeitos principais e as interações entre fatores através do programa Statistica, versão 6.0.

Todos os ensaios foram submetidos a testes sensoriais de Comparação Múltipla, utilizando uma escala hedônica de 7 pontos, em um delineamento de blocos incompletos inteiramente casualizados. Foram recrutados 12 provadores, entre professores, alunos e funcionários do Departamento de Nutrição da Universidade Federal de Pernambuco.

Os dados da análise sensorial foram avaliados através da análise de variância (ANOVA), sendo aplicado o Teste de Duncan a 5\%.

Os produtos obtidos nos ensaios foram avaliados logo após o processamento, e com intervalo de 30, 60, e 90 dias de acordo com a PORTARIA № 451[7], quanto a bolores e leveduras foram avaliados através de técnica padrão [2].

O fruto in natura e o produto preferido foram ainda avaliados segundo RDC. $n^{\circ} 12 / 01$ [8], quanto a coliforme a $45^{\circ} \mathrm{C}$ e Salmonella sp segundo técnica padrão [2].

\section{3 - RESULTADOS E DISCUSSÃO}

\section{1- Planejamento fatorial $2^{3}$}

As respostas obtidas a partir dos dados gerados através dos ensaios tecnológicos, encontram-se apresentados nas Tabelas 3 e 4 onde se constata que a variação de peso da fruta foi negativo, face ao ganho de soluto que superou a perda de água, discordando dos achados de diversos autores, que citam a perda de peso como uma das vantagens do processo $[1,4,5,10,15,16,23,34]$. Esta resposta, que foi influenciada de modo significativo pela concentração do agente osmótico (Tabela 4), também pode ser decorrente da perda de liquidos do interior das células durante a operação de corte da fruta, pois segundo RAVEN et al [31], os vacúolos, espaços presentes no interior das células, contêm o denominado suco celular, cujo principal constituinte é a água. O tamanho e forma da partícula, de acordo com KROKIDA et al [21], também devem ser considerados face aos resultados dos estudos de cinética realizados por LERICI et al [23], que relatam maior ganho de açúcar para maçãs em cubo do que na forma de fatias.

Outra hipótese plausivel estaria relacionada à acidificação do agente osmótico que, segundo Atkinson et al (1952) e Grosso (1972) citados por RODRIGUES e BILHALVA [33], pode ter acarretado um aumento da porosidade do tecido da fruta, facilitando a difusão dos açúcares para o seu interior.

TABELA 3. Efeitos dos fatores utilizados na matriz de planejamento sobre variáveis pré-estabelecidas.

\begin{tabular}{cccc}
\hline ENSAIO & VP (\%) & PU (\%) & IS (\%) \\
\hline 1 & $-19,57$ & 20,93 & 41,52 \\
2 & $-29,40$ & 32,52 & 65,67 \\
3 & $-17,28$ & 20,92 & 39,59 \\
4 & $-31,19$ & 33,00 & 65,71 \\
5 & $-15,34$ & 19,90 & 37,51 \\
6 & $-26,71$ & 35,00 & 63,50 \\
7 & $-15,35$ & 22,00 & 38,50 \\
8 & $-30,00$ & 40,00 & 70,70 \\
9 & $-25,08$ & 30,14 & 56,14 \\
10 & $-23,24$ & 30,96 & 55,98 \\
11 & $-22,00$ & 29,46 & 54,22 \\
\hline
\end{tabular}

Sendo: $\mathrm{VP}$ = variação de peso; $\mathrm{PU}$ = perda de umidade; $\mathrm{IS}$ = GA = incorporação de sólidos ou ganho de açúcar.

TABELA 4. Efeitos dos fatores principais ('Brix, Tipo de açúcar e Temperatura) e de suas interações sobre as respostas obtidas.

\begin{tabular}{cccc}
\hline Fator & Incorporação de sólidos & Perda de Umidade & Variação de peso \\
\hline (1) ${ }^{\circ}$ BRIX & $27,11500^{\star}$ & $14,19250^{\star}$ & $-12,4400^{*}$ \\
(2) AÇÚCAR & 1,57500 & 1,89250 & $-0,7000$ \\
(3) TEMPERATURA & $-0,57000$ & $2,38250^{\star}$ & 2,5100 \\
1 e 2 & 2,04500 & 0,84750 & $-1,8400$ \\
1 e 3 & 1,98000 & $2,35750^{*}$ & $-0,5700$ \\
2 e 3 & 2,52000 & 1,65750 & $-0,9500$ \\
1,2 e 3 & 1,06000 & 0,60250 & 0,2000 \\
\hline
\end{tabular}

* Efeitos significativos

O pré-tratamento com metabissulfito e o branqueamento, podem ter, também, conforme HENG et al [18]; ISLAM e FLINK [19] e PONTING et al [29], favorecido o ganho de sólidos, por aumentar a permeabilidade, diminuindo conseqüentemente a seletividade, e permitindo uma transferência de massa mais rápida.

Neste contexto, o fator temperatura não deve ser esquecido, uma vez que temperaturas acima de $60^{\circ} \mathrm{C}$, provavelmente, afetam as propriedades do tecido das frutas, favorecendo o fenômeno de impregnação, ou seja, o ganho de açúcar [6, 11, 18, 22, 37]. De acordo com Lenart e Lewicki (1990 a) apud SILVA [34], embora a temperatura crítica dependa da natureza da fruta, este fenômeno, assim como o aumento na perda de umidade, poderá ocorrer entre 30 a $90^{\circ} \mathrm{C}$.

No que diz respeito à perda de umidade, além da temperatura constata-se ainda a influência da concentração do agente osmótico, ratificando MOY et al [26] (Tabela 4). A Figura 1 demonstra que a elevação da temperatura acarreta aumento da perda de umidade, que é mais acentuada para a concentração de $70^{\circ}$ Brix $(-0,03$ 
contra -4,74). A interação entre a temperatura e o ${ }^{\circ}$ Brix foi significativa no que se refere à perda de umidade (Tabela 4), conforme já referido para kiwis [37], maçãs [25, 28], banana [6], manga e mamão [26], e abacaxi [30].

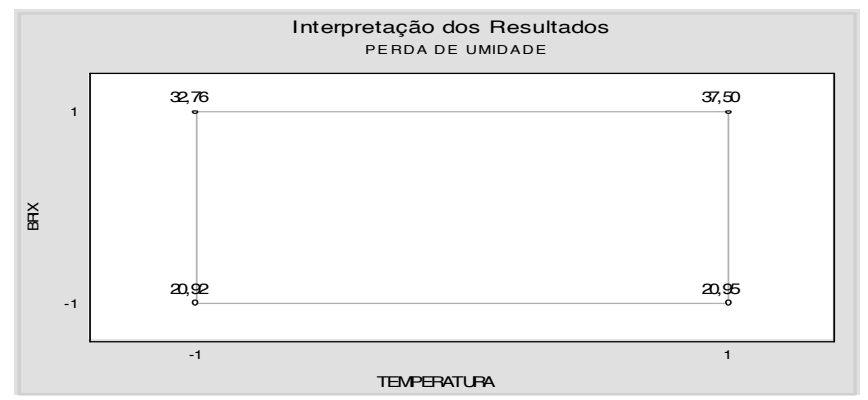

FIGURA 1. Interpretação dos Resultados (Perda de Umidade).

Observando a Tabela 4, constata-se que o aumento da concentração exerceu efeito significativo para o ganho de sólidos, acordando com BERISTAIN et al [4], MAGEE et al [27] e RAHMAN e LAMB [32].

Com vistas a reduzir a incorporação de solutos, sugere-se que sejam testadas outras formas de corte, como exemplo: em fatias que, além de facilitar esta operação, permitirá uma maior uniformidade dos pedaços [25] e o emprego de uma cobertura comestivel de alta afinidade pela água e baixa afinidade pelo soluto de modo a reduzir sua incorporação. Neste contexto os polissacarídeos se apresentam como os compostos mais adequados, especialmente os alginatos e as pectinas pela baixa metoxilaçao por formarem géis iônicos através de ligações cruzadas com os ions $\mathrm{Ca}^{+2}[1]$.

\section{2 - Avaliação da estabilidade dos produtos obtidos}

Os produtos foram avaliados por um período de 90 dias durante o qual permaneceram estocados sob temperatura ambiente de $28^{\circ} \mathrm{C} \pm 3^{\circ} \mathrm{C}$. Do ponto de vista microbiológico, com exceção do produto $\mathrm{n}^{\circ} 5$, que não apresentou conformidade com o padrão [7], as contagens de Bolores e Leveduras foram inferiores a $90 \mathrm{UFC} / \mathrm{g}$, comprovando os efeitos benéficos do processamento.

Estes resultados motivaram a substituição do produto $\mathrm{n}^{\circ} 5$ pelo fruto in natura na realização dos testes sensoriais, com vistas a avaliar os efeitos dos diferentes tratamentos utilizados sobre as características organolépticas do jenipapo. Os resultados obtidos, Tabelas 5 e 6 demonstraram que todos os produtos diferiram do fruto in natura, embora de modo não significativo $(p<0,05)$, provavelmente devido à elevada incorporação de soluto que os tornaram mais doces, sabor bastante apreciado na região nordeste.

Através do percentual do somatório das notas atribuidas para cada produto, Figura 2, observa-se que ao 9 foram conferidas as maiores notas, no que foi seguido em ordem decrescente pelo 2 e 4 . O produto preferido (ponto central) foi também avaliado quanto à presença de Salmonella sp em $25 \mathrm{~g}$ e coliforme a $45^{\circ} \mathrm{C} / \mathrm{g}$, que se apresentaram ausentes. Comparando os percentuais destes produtos com os do fruto fresco verifica-se uma considerável diferença entre os mesmos demonstrando que a negativa variação de peso e a elevada incorporação de sólidos favoreceram a aceitabilidade dos produtos. Como característica comum, produtos supra citados apresentam uma perda de umidade de 30,14\%, $32,52 \%$ e $33,00 \%$, respectivamente, que pode ser considerada satisfatória. Este resultado permite inferir que estes produtos foram preferidos pelos juizes. Diante destas evidências a aplicação desta tecnologia parece constituir uma boa alternativa para aumentar o consumo deste fruto, face à sua restrita aceitabilidade in natura.

TABELA 5. Resultado da Avaliação Sensorial.

\begin{tabular}{|c|c|c|c|c|c|c|c|}
\hline \multirow[t]{2}{*}{ BLOCO } & \multicolumn{6}{|c|}{ REPETIÇÃ̃O } & \multirow{2}{*}{$\begin{array}{l}\text { BLOCO } \\
\text { TOTAL }\end{array}$} \\
\hline & Produtos & Notas & Produtos & Notas & Produtos & Notas & \\
\hline 1 & (1) & 5 & (2) & 6 & (3) & 3 & 14 \\
\hline 2 & (4) & 5 & $(5)^{*}$ & 2 & (6) & 6 & 13 \\
\hline \multirow[t]{2}{*}{3} & (7) & 6 & (8) & 7 & (9) & 6 & 19 \\
\hline & & & & & & & 46 \\
\hline 4 & (1) & 5 & (4) & 6 & (7) & 2 & 13 \\
\hline 5 & (2) & 6 & $(5)^{*}$ & 2 & (8) & 6 & 14 \\
\hline \multirow[t]{2}{*}{6} & (3) & 5 & (6) & 2 & (9) & 7 & 14 \\
\hline & & & & & & & 41 \\
\hline 7 & (1) & 4 & $(5)^{*}$ & 3 & (9) & 6 & 13 \\
\hline 8 & (4) & 7 & (8) & 3 & (3) & 5 & 15 \\
\hline \multirow[t]{2}{*}{9} & (7) & 5 & (6) & 6 & (2) & 6 & 17 \\
\hline & & & & & & & 45 \\
\hline 10 & (3) & 6 & $(5)^{*}$ & 2 & (7) & 7 & 15 \\
\hline 11 & (6) & 6 & (8) & 4 & (1) & 3 & 13 \\
\hline \multirow[t]{2}{*}{12} & (9) & 7 & (4) & 5 & (2) & 6 & 18 \\
\hline & & & & & & & 46 \\
\hline
\end{tabular}

* O produto 5, por apresentar UFC/g de Bolores e Leveduras acima do permitido pela Portaria $n^{\circ} 451$, foi substituído pelo fruto in natura.

TABELA 6. Análise da variância dos dados da Análise Sensorial.

\begin{tabular}{ccccccc}
\hline Fontes de variação & $\begin{array}{c}\text { Grau de } \\
\text { Liberdade }\end{array}$ & $\begin{array}{c}\text { Soma } \\
\text { Quadrática }\end{array}$ & $\begin{array}{c}\text { Média } \\
\text { Quadrática }\end{array}$ & Fcalc. & F5\% \\
\hline Total & 35 & 95,89 & & & \\
Repetições & 3 & 1,89 & 0,63 & 0,28 & 3,24 \\
Blocos (repetições) & 8 & 14,00 & 1,75 & 0,78 & 2,59 \\
Experimentos & 8 & 44,15 & 5,52 & 2,46 & 2,59 \\
Resíduo & 16 & 35,85 & 2,24 & & \\
\hline
\end{tabular}

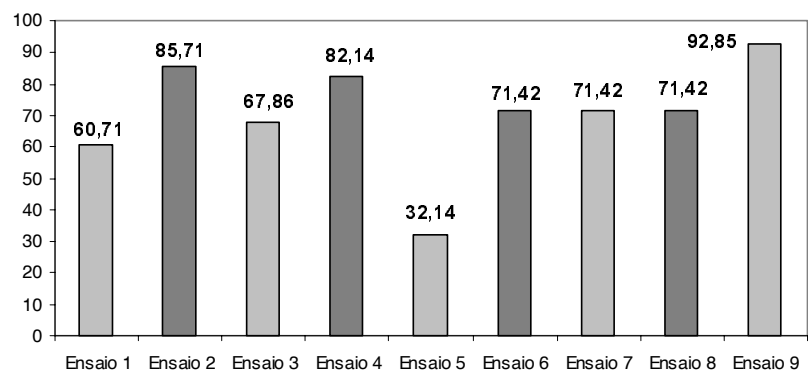

Ensaios

FIGURA 2. Percentual do somatório das notas atribuídas aos produtos resultantes dos ensaios realizados. 
Além de contribuir para melhoria das caracteristicas organolépticas, constata-se, através de uma comparação entre a composição da matéria-prima e o produto preferido, do ponto de vista nutricional, que o processo propiciou um aumento do valor energético do Jenipapo (Figura 3).

Do ponto de vista econômico, pode-se ressaltar o baixo custo do agente osmótico que apresenta a possibilidade de reutilização do mesmo e o baixo custo de energia, uma vez que os experimentos mais aceitos foram àqueles implementados a $60^{\circ} \mathrm{C}$. Os custos adicionais, relativos a utilidades e embalagem serão estimados após otimização de processamento e determinação do prazo de validade do produto.

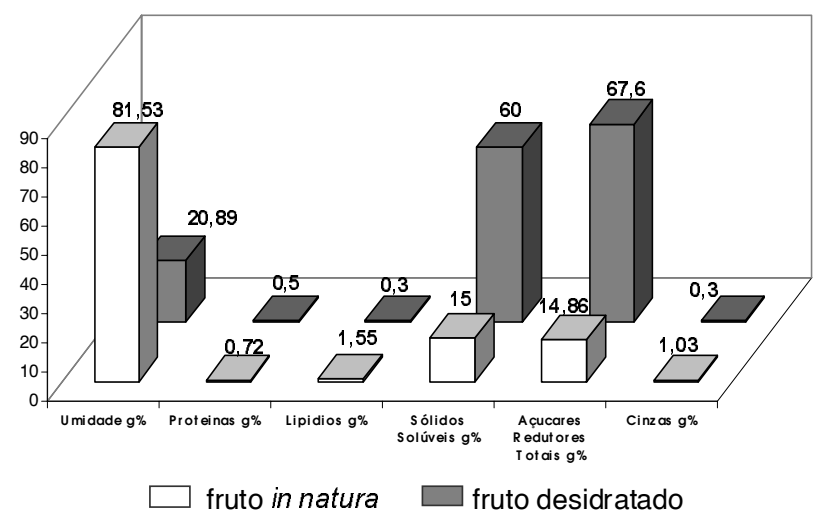

FIGURA 3. Efeitos da desidratação osmótica sobre a composição físico-química.

\section{4 - CONCLUSÕES}

Os resultados obtidos permitem concluir que:

- o processo de desidratação osmótica aplicado ao jenipapo permitiu a obtenção de um produto com satisfatória aceitabilidade e estabilidade microbiológica;

- embora as melhores respostas, em relação à perda de umidade, tenham sido obtidas nas condições de processamento a $70^{\circ} \mathrm{C}$, açúcar cristal como agente osmótico na concentração de $70^{\circ} \mathrm{Brix}$, o produto obtido, nas condições do ponto central (açúcar cristal e demerara, $60^{\circ} \mathrm{C}$ e $60^{\circ}$ Brix) foi o preferido.

- o comportamento observado com relação à incorporação de soluto, que implicou em aumento de peso, encontra-se associado às características próprias do jenipapo; deverá ser objeto de pesquisa com vistas à otimização do produto.

\section{5 - REFERÊNCIAS}

[1] AZEREDO, H. M. C.; JARDINE, J. G. Desidratação Osmótica de Abacaxi aplicada à Tecnologia de Métodos combinados. Ciênc. Tecnol. Aliment., Campinas, v.20,n. 1,p.7482, jan./abr.,2000.

[2] A. O. A. C. Association of Official Analytical Chemists: Official Methods of Analysis 16. ed. Washington, 1998.

[3] BARRETREINA, L. del C.; CHITARRA, M. I. F.; CHITARRA, A. B. Choque a frio e Atmosfera modificada no aumento da vida pós-colheita de tomates. 1- Avaliação da Qualidade. Ciênc. Tecnol. Aliment., Campinas, v.13,n.2,p.168-183, jul/dez., 1993.

[4] BERISTAIN, C. I.; AZUARA, E.; CORTÉZ, R.; GARCIA, H. S. Mass Transfer during osmotic dehydration of pineapple rings. International Journal of Food Science and Technology, Glasgow, v.25, n.5, p.576-582, 1990.

[5] BOLIN, H. R.; HUXSOLL, C. C.; JACKSON, R. and NG, K. C. Effect of Osmotic Agents and Concentration on Fruit Quality. Journal of Food Science, V.48, p.202-205, 1983.

[6] BONGIRWIR, D. R.; SREENIVASAN, A. Studies on osmotic dehydration of bananas. Journal of Food Science and Technology, India, v. 14, n.3.p. 104-112, 1977.

[7] BRASIL. Portaria $n^{\circ}$ 451, de 19 de Setembro de 1997. Aprova o regulamento técnico e princípios gerais para o estabelecimento de critérios e padrões microbiológicos para alimentos. D.O. U. [Diário Oficial da União]. Brasília, 02 de julho de 1998.

[8] BRASIL. Ministério da Saúde. Secretaria Nacional de Vigilância Sanitária de Alimentos. Resolução n. 12/01. Regulamento Técnico sobre Padrões Microbiológicos para Alimentos. In: Diário Oficial, [da Republica Federativa do Brasil]. Brasília, 02 de janeiro, 2001.

[9] CAVAlCANTE, D. P. Frutas Comestíveis da Amazônia II. Publicações avulsas no 27, Belém, 1974.

[10] FALCONE, M. A. ; SUAZO, C. A.T. Desidratação osmótica do abacaxi (Ananás comosus, (L.) Merril). Parte I: Influência da temperatura e concentração do xarope sobre a velocidade de secagem. Boletim da SBCTA, Campinas, v.22,n.1/2, p.17-35, jan/jun 1988.

[11] FARKAS, D. F.; LAZAR, M. E. Osmotic dehydration of apple pieces: effect of temperature and syrup concentration on rates. Food Technology, Chicago, v.23, p.688-90, 1969.

[12] FIGUEIREDO, R. W. Estudo da Industrialização do jenipapo (Genipa americana L.). Fortaleza, 1984. 171p. Tese (Mestrado em Tecnologia de Alimentos) - Universidade Federal do Ceará.

[13] FIGUEIREDO, R. W. Avaliação da Preservação da Polpa de jenipapo (Genipa americana L.) por alta e baixa temperatura. Ciênc. Agron., Fortaleza, v. 17, n.2,p.65-73, Dez., 1986.

[14] FIgUeIREDO, R. W.; MAIA, G. A.; MONTERO, J. C. S.; FIGUEIREDO, E. A. T. Ácidos Graxos na fração lipídica da polpa e semente do jenipapo (Genipa americana L.) B. CEPPA, Curitiba, v.9,n.2,p.149-154, jul./dez.,1991.

[15] GARROTE, R. L.; SILVA, E. R.; BERTONE, R. A . Osmotic concentration at $5^{\circ} \mathrm{C}$ and $25^{\circ} \mathrm{C}$ of pear and apple cubes and strawberry halves. Lebensmittel-Wissenschaft undTechnologie, London, v.25, n.2, p.133-138, 1992.

[16] GIANGIACOMO, R.; TORREGIANI, D.; ABBO, E. Osmotic dehydration of fruit: Part 1. Sugars exchange between fruit and extracting syrups. Journal of Food Processing and Preservation, Westport, v. 11, n.3, p. 183-195, 1987.

[17] GOMES, R. P. Fruticultura Brasileira. 11 ed. São Paulo: Nobel, 1989.p.278-281.

[18] HENG, K.; GUILBERT, S.; CUQ, J.L. Osmotic dehydration of papaya: influences of process variables on the product quality. Science des Aliments, Paris, v. 10, n.4, p.831848, 1990.

[19] ISLAM, M.N.; FLINK, J. N. II. Osmotic concentration and its effect on air drying behaviour. Journal Food Technology, Chicago, v.17, p.387-403, 1982.

[20] KARATHANOS, V. T.; KOSTAROPOULOS, A. E. Air-drying Kinetics of osmotically dehydrated fruits. Drying 
Technology, New York, v.13, n.5-7, p.1503-152 1, 1995.

[21] KROKIDA, M. K.; KIRANOUDIS, C. T.; MAROULIS, Z. B.; MARINOS - KOURIS, D. Drying related Properties of apple. Drying Technology, New York, v. 18, n.6, p. 12511267, 2000.

[22] LARANJEIRA, H.C.A . Otimização do processo de Desidratação Osmótica de Abacaxi (Ananás comosus (L.) Merril) para aplicação à Tecnologia de Métodos Combinados. Campinas, 1997. 100p. Dissertação (Mestre em Engenharia de Alimentos) - Universidade Estadual de Campinas.

[23] LENART, A.; FLINK, J.M. Osmotic concentration of potato. II. Spatial distribution of the osmotic effect. Journal Food Technology, Chicago v.19, p.65-89, 1984.

[24] LERICI, C. R.; PINNAVAIA, M.; DALLA ROSA, M.; BARTOLUCCI, L. Osmotic Dehydration of Fruit: Influence of Osmotic Agents on Drying Behavior and Product Quality. Journal of Food Science, Chicago, v.50, p. $1217-1219,1985$.

[25] LIU, H. A Kinetic study of salt diffusion in potato at high temperature. International Journal of Food Science an Technology, Wolfville, v.27, p, 443-455, 1992.

[26] MAGEE, T. R. A.; HASSABALLAH, A. A.; MURPHY, W.R. Ir. Journal Food Science and Technology, v.7, p. 147-155, 1983.

[27] MOY, J.H.; LAU, N.B.H.; DOLLAR, A.M. Effects of sucrose and acids on osmovac-dehydration of tropical fruits. Journal of Food Processing and Preservation, Westport, v.2, n.2, p.131-135, 1978.

[28] NETO, B.B.;SCARMINIO,I.S.;BRUNS,R.E. Como fazer experimentos: pesquisa e desenvolvimento na ciência e na indústria. Campinas, São Paulo: Editora Unicamp, $2001.401 \mathrm{p}$.
[29] POINTING, J. D.; Watters, G. G.; FORREY, R. R.; JACKSON, R.; STANLEY, W. L. Osmotic dehydration of fruits. Food Technology, Chicago, v.20, n.10, p.1365-1368, 1966.

[30] POINTING, J. D. Osmotic Dehydration of Fruits - Recent Modifications and Applications. Process Biochemistry, Watford, v.8, n.12, p.18-20, 1973.

[31] RAHMAN, M.S.; LAMB, J. Osmotic dehydration of pineapple. Journal of Food Science and Technology, Mysore, v.27, n.3, p. 150-152, 1990.

[32] RAVEN, P. H.; EVERT, R.F.; EICHHORN, S. E. Biologia Vegetal. 5 ed. Editora Guanabara Koogan, p. 14-67, 1996.

[33] RAOULT-WACK, A. L. Recent advances in the osmotic dehydration of foods. Trends in Food Science \& Technology, Cambridge, v.5, n.8, p. 255 - 260,1994.

[34] RODRIGUES, R. S. ; BILHALVA, A. B. Saturação de Figo (Fícus carica, L.) com Açucares: influência do $\mathrm{pH}$ e da temperatura. Bol. SBCTA, v.30, n. 1, p. $78-81$, jan/ jun, 1996.

[35] SILVA, S.F. Desidratação Osmótica do Mamão Formosa. Natal, 1998 74p. Tese (Mestre em Engenharia Química) - Universidade Federal do Rio Grande do Norte - UFRN.

[36] SUDENE. "Valor Nutritivo e o Aproveitamento Industrial das Frutas Regionais" Recife, 1971. (Relatório final do Convênio SUDENE/Ministério da Agricultura).

[37] TORREGIANI, D. Osmotic dehydration in fruit and vegetable processing. Food Research International, London, v.26, n. 1, p.59-68, 1993.

[38] VIAL. C.; GUILBERT, S.; CUQ, J. Osmotic dehydration of kiwi Fruits: Influence of Process Variables on the color and Acid content. Sciences des Aliments, Paris, v.11, n. 1, p.63-84, 1991. 